\title{
Método de localización de fallas basado en impedancia aparente para sistemas de distribución con generación distribuida
}

\author{
Impedance-based fault location method for power distribution \\ system considering distributed generation
}

\author{
César A. Orozco Henao $\quad$ Juan José Mora Flórez ${ }^{2} \quad$ Sandra M. Pérez Londoño ${ }^{2}$ \\ Recibido 12 de junio de 2012, aceptado 6 de octubre de 2014 \\ Received: June 12, 2012 Accepted: October 6, 2014
}

\begin{abstract}
RESUMEN
La metodología propuesta en este artículo resuelve, de manera sencilla, el problema de localización de fallas en sistemas de distribución con generación distribuida (GD). Su practicidad radica en la estimación de las corrientes que alimentan la falla a partir de las mediciones de tensiones y corrientes registradas en la subestación y en el nodo donde se encuentra la GD, esto evita la utilización de los parámetros y el modelado de los generadores, que para la mayoría de los casos no están disponibles. A su vez, la utilización de las medidas registradas en los centros de generación del sistema permite considerar cualquier variación presente en los estados de falla y prefalla. La propuesta presentada en este artículo consiste en un método de localización de fallas basado en la estimación de la impedancia, que considera el efecto de la generación distribuida y la variación de la corriente de carga, a partir de las mediciones de tensiones y corrientes registradas en los centros de generación. La metodología propuesta se valida en el sistema IEEE 34 nodos, el que se modifica conectando un nuevo generador en el radial más largo. En este nuevo sistema se simulan fallas monofásicas, bifásicas y trifásicas, considerando resistencias de falla entre $0 \Omega$ y $40 \Omega$ y contribución de generación distribuida entre $5 \%$ y $50 \%$. Los resultados obtenidos son superficies de desempeño, estas presentan errores de estimación inferiores a $2 \%$.
\end{abstract}

Palabras clave: Localización de fallas, generación distribuida, estimación de carga, calidad de potencia, sistemas de distribución de energía eléctrica.

\begin{abstract}
This work aims to solve the problem of fault location considering distributed generation (DG). As one of most relevant characteristics of this proposed method, the fault current is estimated using the measurements of voltage and current obtained at the power substation and at the node where the DG is placed. These last measurements avoid the effort of using an electrical model to consider the impact of the distributed generator and it also makes possible to take into account their variations in fault state and pre-fault state. This methodology is based on the estimation of the fault impedance, which considers the effect of distributed generation and the variation of load current. The proposal is validated under the IEEE 34-nodes test system, which is modified to connect one generator in the longest radial. Tests are performed considering single phase, phase to phase and three phase faults, and resistances between 0 and $40 \mathrm{ohms}$ and distributed generation contribution between $5 \%$ and $50 \%$. The results obtained are performance surfaces, which show errors bellow $2 \%$ at the estimation of the fault distance.
\end{abstract}

Keywords: Fault location, distributed generation, load estimation, power quality, electric power distribution system.

\footnotetext{
1 Departamento de Engenharia elétrica. Universidade Federal do Rio Grande do Sul. Brasil. E-mail: orozco.henao@ufrgs.br

2 Facultad de ingeniería eléctrica. Universidad Tecnológica de Pereira. Colombia. E-mail: jjmora@utp.edu.co; saperez@utp.edu.co
} 


\section{INTRODUCCIÓN}

Los grandes avances tecnológicos logrados en las últimas décadas han motivado el desarrollo de herramientas y estrategias que permitan el uso eficiente de la energía. Esta mirada abre nuevos desarrollos de innovación pensados en la creación de sistemas inteligentes que permitan controlar e introducir nuevas características a los sistemas eléctricos convencionales. Dichas características están asociadas al control inteligente de la demanda y el ingreso de pequeños centros de generación con fuentes alternativas. Estos avances son incluidos en el concepto de las smart grids que no es más que la búsqueda del gerenciamiento inteligente de los sistemas eléctricos para el uso eficiente de la energía [1]. El concepto de las smart grids ha tomado mayor fuerza sobre los sistemas de distribución de energía eléctrica debido a la aparición de pequeños centros de generación distribuida que se presenta con gran frecuencia, debido a los avances obtenidos en el desarrollo de las fuentes de energías renovables y a la concientización de la sociedad en el uso de fuentes de energías limpias. No obstante, esto introduce un problema en los sistemas de control y protección, debido a la aparición de corrientes bidireccionales, lo que incide en la calidad del servicio [2].

Un problema asociado a la calidad es la continuidad de suministro. Esta se ve afectada por la ocurrencia de fallas en el sistema de distribución y tradicionalmente ha sido abordada sin considerar el efecto de la generación distribuida [3-6]. Recientemente, algunas metodologías se han propuesto para considerar el problema de localización de fallas en sistemas de distribución con GD. Bretas y Salim en [7-8] presenta un primer acercamiento con un método de localización de fallas que utiliza el modelo de un generador síncrono para representar el efecto de la GD. Sin embargo, la metodología carece de robustez, debido a que no considera variaciones en el sistema. A partir de este trabajo, Nunes y Breatas presenta en [9-10] una estrategia interesante que introduce el efecto de GD en la corriente de carga, para ello utiliza el modelo del generador síncrono, siendo necesario el conocimiento de sus parámetros. Un concepto diferente es presentado por Marvik, Petterteig y Hoidalen en [11-12], donde se muestra una estrategia de localización que introduce el efecto de la generación distribuida por medio de dos factores de corrección. No obstante, esta metodología utiliza las reactancias subtransitorias y las tensiones internas de los generadores, ello obliga a tener conocimiento de los parámetros del generador que en muchos casos no se conocen con precisión.

En la propuesta que se presenta en este artículo se considera el efecto de la GD en la estimación de la corriente que alimenta a la falla desde la fuente GD. Esta corriente se calcula a partir de las mediciones de tensión y corriente registradas en las unidades de generación, esto evita la utilización del modelo y los parámetros del generador. Adicionalmente se consideran las variaciones en la carga para implementar un método robusto de localización de fallas. Lo anterior hace que esta sea una alternativa sencilla y fácil de aplicar en sistemas de distribución reales.

Como contenido de este artículo se presenta el desarrollo matemático de la metodología propuesta. Seguidamente se presenta el algoritmo propuesto para el método de localización de fallas; luego se presentan las pruebas y los resultados obtenidos en un sistema de distribución prototipo. Finalmente, se presentan las conclusiones más importantes asociadas a la investigación realizada.

\section{MÉTODO PROPUESTO PARA LA LOCALIZACIÓN DE FALLAS}

Para la definición del método propuesto se asume un alimentador de distribución como el mostrado en la Figura 1, conformado por $x$ ramificaciones o radiales. Se considera que solo se tienen medidas de tensión y corriente en la subestación y en la fuente GD. También, que el alimentador tiene una falla entre el nodo $k$ y el $k+1$.

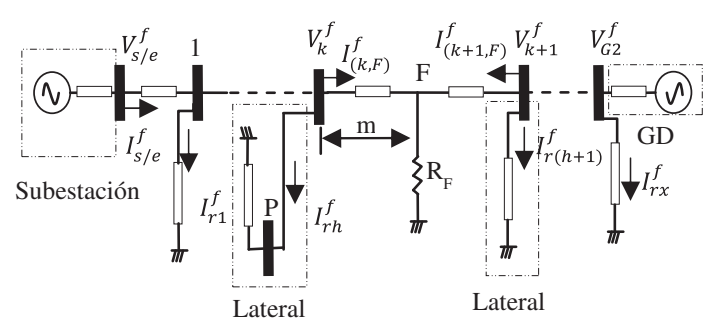

Figura 1. Modelo del sistema en estudio bajo falla.

De la Figura 1, las variables para circunstancias de falla indicadas con el superíndice $f$ son: 
$V_{\text {s/e }}^{f}$ : Tensión en la cabecera del circuito.

$I_{s / e}^{f}$ : Corriente en la cabecera del circuito.

$V_{G 2}^{f}$ : Tensión de la generación distribuida.

$I_{G 2}^{f}$ : Corriente de la generación distribuida.

$V_{k}^{f}: \quad$ Tensión en el nodo $k$.

$I_{(k, F)}^{f}$. Corriente de fase desde el nodo $k$ hacia el $I_{(k+1, F)}^{f}$ Corriente de fase desde el nodo $(k+1)$

$I_{(k+1, F)}^{f}$ : hacia el nodo $F$ en falla.

$I_{r h}^{f}$ : Corriente que se deriva por el ramal $h$.

$I_{F}$ : Corriente por la resistencia de falla.

A partir de los registros de tensión y corriente en la subestación se calcula la tensión y la corriente para todas las secciones que conforman el alimentador. Una vez obtenidas las corrientes y las tensiones en todos los nodos se puede reducir el análisis de cortocircuito exclusivamente al tramo donde ocurrió la falla, como se muestra en esta sección [13].

\section{Análisis para falla monofásica}

Se asume la falla monofásica entre los nodos $k$ y $k+1$, modelada como se muestra en la Figura 2.

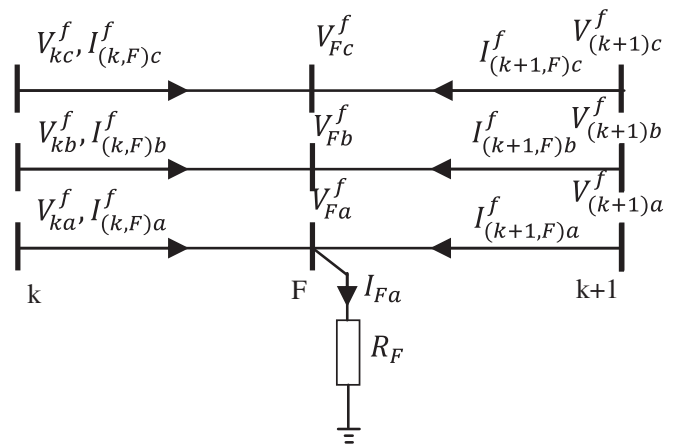

Figura 2. Modelo equivalente de una falla monofásica.

A partir del análisis circuital del modelo mostrado en la Figura 2 se obtiene el sistema de ecuaciones (1), para las fases $i(i=a, b, c)$.

$$
\left[\begin{array}{c}
V_{k a}^{f} \\
V_{k b}^{f} \\
V_{k c}^{f}
\end{array}\right]=\left[\begin{array}{c}
\Delta V_{a-F} \\
\Delta V_{b-F} \\
\Delta V_{c-F}
\end{array}\right]+\left[\begin{array}{ccc}
R_{F} & 0 & 0 \\
0 & 0 & 0 \\
0 & 0 & 0
\end{array}\right]\left[\begin{array}{c}
I_{(k+1, F) a}^{f} \\
0 \\
0
\end{array}\right]
$$

Donde las variaciones en la tensión están dadas por (2).

$$
\left[\begin{array}{c}
\Delta V_{a-F} \\
\Delta V_{b-F} \\
\Delta V_{c-F}
\end{array}\right]=\left[\begin{array}{ccc}
m Z_{a a}+R_{F} & m Z_{a b} & m Z_{a c} \\
m Z_{b a} & Z_{b b} & Z_{b c} \\
m Z_{c a} & Z_{c b} & Z_{c c}
\end{array}\right]\left[\begin{array}{c}
I_{(k, F) a}^{f} \\
I_{(k, F) b}^{f} \\
I_{(k, F) c}^{f}
\end{array}\right]
$$

Donde,

$m$ : Distancia a la falla en por unidad de longitud.

$Z_{i i}$ : Impedancia propia de la línea para la fase $i$ con $i=(a, b, c)$, en el tramo en falla.

$Z_{i j}$ : Impedancia mutua de la línea entre fases $i$ y $j$, en el tramo en falla $(i \neq j)$.

De la primera fila del sistema de ecuaciones (1) se obtiene la ecuación (3), en función de las dos variables de interés: la resistencia de falla $\left(R_{F}\right)$ y la distancia a la falla $(\mathrm{m})$.

$$
V_{k a}^{f}=\Delta V_{a-F}+R_{F} \cdot\left(I_{(k, F) a}^{f}+I_{(k+1, F) a}^{f}\right)
$$

Si se divide la ecuación (3) por $\Delta V_{a}^{f}=Z_{a a}$. $I_{(k, F) a}^{f}+Z_{a b} \cdot I_{(k, F) b}^{f}+Z_{a c} \cdot I_{(k, F) c}^{f}$, con la parte imaginaria se encuentra una expresión para el cálculo de la resistencia de falla, como se muestra en la ecuación (4).

$$
R_{F}=\frac{\operatorname{imag}\left\{\frac{V_{k a}^{f}}{\left.\Delta V_{a}^{f}\right\}}\right.}{\operatorname{imag}\left\{\frac{I_{(k, F) a}^{f}+I_{(k+1, F) a}^{f}}{\Delta V_{a}^{f}}\right\}}
$$

Con la resistencia de falla, en la ecuación (3) se puede obtener la distancia a la falla $(\mathrm{m})$ como se presenta en la ecuación (5).

$$
m=\frac{V_{k a}^{f}-R_{F} \cdot\left(I_{(k, F) a}^{f}+I_{(k+1, F) a}^{f}\right)}{Z_{a a} \cdot I_{(k, F) a}^{f}+Z_{a b} \cdot I_{(k, F) b}^{f}+Z_{a c} \cdot I_{(k, F) c}^{f}}
$$

\section{Análisis para fallas bifásicas}

Se asume la falla bifásica entre los nodos $k \mathrm{y} k+1$, modelada como se muestra en la Figura 3.

Igual que para la falla monofásica, se realiza un análisis circuital del modelo mostrado en función de la tensión en la resistencia del suelo $\left(V_{g}^{f}\right)$. Esto 


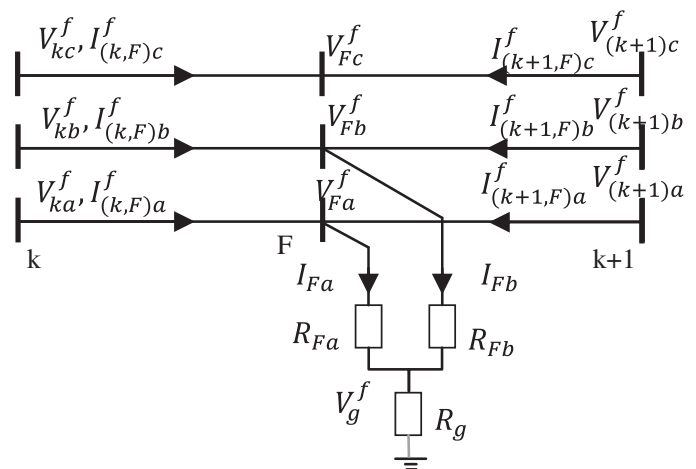

Figura 3. Modelo equivalente de una falla bifásica.

se efectúa con el fin de formular un análisis general para las fallas bifásicas y bifásicas a tierra. El sistema de ecuaciones del modelo general se presenta en la ecuación (6).

$\left[\begin{array}{c}V_{k a}^{f}-V_{g}^{f} \\ V_{k b}^{f}-V_{g}^{f} \\ V_{k c}^{f}\end{array}\right]=\left[\begin{array}{c}\Delta V_{a g-F} \\ \Delta V_{b g-F} \\ \Delta V_{c g-F}\end{array}\right]+\left[\begin{array}{ccc}R_{F} & 0 & 0 \\ 0 & R_{F} & 0 \\ 0 & 0 & 0\end{array}\right]\left[\begin{array}{c}I_{(k+1, F) a}^{f} \\ I_{(k+1, F) b}^{f} \\ 0\end{array}\right](6)$

Donde las variaciones en la tensión están dadas por (7).

$\left[\begin{array}{c}\Delta V_{a g-F} \\ \Delta V_{b g-F} \\ \Delta V_{c-F}\end{array}\right]=\left[\begin{array}{ccc}m Z_{a a}+R_{F} & m Z_{a b} & m Z_{a c} \\ m Z_{b a} & m Z_{b b}+R_{F} & m Z_{b c} \\ m Z_{c a} & m Z_{c b} & Z_{c c}+Z_{l c}\end{array}\right]\left[\begin{array}{c}I_{(k, F) a}^{f} \\ I_{(k, F) b}^{f} \\ I_{(k, F) c}^{f}\end{array}\right]$ (7)

De las filas uno y dos del sistema de ecuaciones (6) se obtienen dos ecuaciones en función de $m, R_{F} \mathrm{y}$ $V_{g}^{f}$. Estas dos ecuaciones se restan para eliminar la incógnita $V_{g}^{f}$, con el fin de encontrar una ecuación general que no dependa si la falla es o no a tierra, como se muestra en (8).

$$
\Delta V_{a b}^{f}=m\left(K_{1} I_{(k, F) a}^{f}+K_{2} I_{(k, F) b}^{f}+K_{3} I_{(k, F) c}^{f}\right)+R_{F} \Delta I_{F a b}^{f}(8)
$$

Donde,

$$
\begin{gathered}
\Delta V_{a b}=V_{k a}^{f}-V_{k b}^{f} \\
\Delta I_{F a b}^{f}=\left[\left(I_{(k, F) a}^{f}+I_{(k+1, F) a}^{f}\right)-\left(I_{(k, F) b}^{f}+I_{(k+1, F) b}^{f}\right)\right] \\
K_{1}=Z_{a a}-Z_{b a} \\
K_{2}=Z_{a b}-Z_{b b} \\
K_{3}=Z_{a c}-Z_{b c}
\end{gathered}
$$

De manera similar como se realiza para la falla monofásica, a partir de la ecuación (8) se determinan dos expresiones para el cálculo de la resistencia de falla $\left(R_{F}\right)$ y la distancia a la falla $(m)$ como se presenta en (10) y (11), respectivamente.

$$
R_{F}=\frac{\operatorname{imag}\left\{\frac{V_{k a}^{f}-V_{k b}^{f}}{\Delta V_{a b}^{f}}\right\}}{\operatorname{imag}\left\{\frac{\Delta I_{F a b}^{f}}{\Delta V_{a b}^{f}}\right\}}
$$

$$
m=\frac{V_{k a}^{f}-V_{k b}^{f}-R_{F} \Delta I_{F a b}^{f}}{\Delta V_{a b}^{f}}
$$

\section{Análisis para fallas trifásicas}

Se considera una falla trifásica entre los nodos $k$ y $k+1$, modelada como se muestra en la Figura 4.

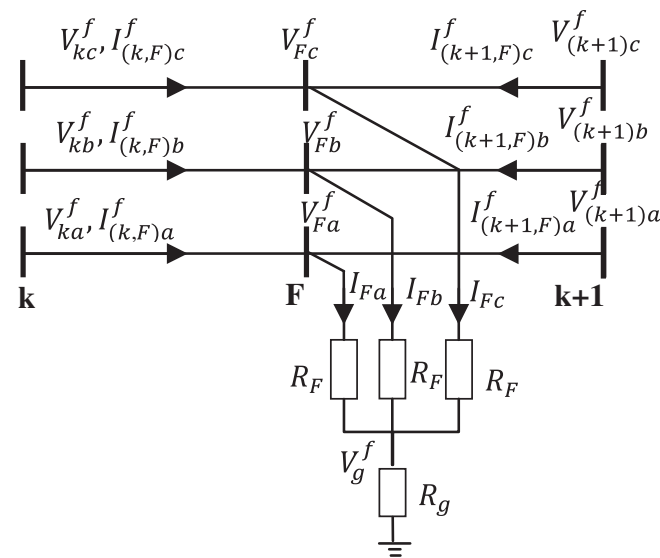

Figura 4. Modelo equivalente de una falla trifásica.

Igual que para los casos anteriores de fallas se busca encontrar un sistema de ecuaciones para representar de manera exacta y general los dos tipos de fallas trifásicas (trifásicas y trifásicas a tierra). Por esta razón, el análisis se realiza en función de la tensión de la resistencia del suelo $\left(V_{g}^{f}\right)$, como se muestra en el sistema de ecuaciones (12).

$\left[\begin{array}{c}V_{k a}^{f}-V_{g}^{f} \\ V_{k b}^{f}-V_{g}^{f} \\ V_{k c}^{f}-V_{g}^{f}\end{array}\right]=\left[\begin{array}{c}\Delta V_{a g-F} \\ \Delta V_{b g-F} \\ \Delta V_{c g-F}\end{array}\right]+\left[\begin{array}{ccc}R_{F} & 0 & 0 \\ 0 & R_{F} & 0 \\ 0 & 0 & R_{F}\end{array}\right]\left[\begin{array}{c}I_{(k+1, F) a}^{f} \\ I_{(k+1, F) b}^{f} \\ I_{(k+1, F) c}^{f}\end{array}\right]$

Donde las variaciones en la tensión están dadas por (13). 
$\left[\begin{array}{c}\Delta V_{a g-F} \\ \Delta V_{b g-F} \\ \Delta V_{c g-F}\end{array}\right]=\left[\begin{array}{ccc}m Z_{a a}+R_{F} & m Z_{a b} & m Z_{a c} \\ m Z_{b a} & m Z_{b b}+R_{F} & m Z_{b c} \\ m Z_{c a} & m Z_{c b} & m Z_{c c}+R_{F}\end{array}\right]\left[\begin{array}{l}f \\ I_{(k, F) a}^{f} \\ I_{(k, F) b}^{f} \\ I_{(k, F) c}^{f}\end{array}\right](13)$

Del sistema de ecuaciones (12) se pueden obtener tres conjuntos de ecuaciones tanto para la resistencia de falla $\left(R_{F}\right)$ como para la distancia de falla $(m)$. Estas se presentan desde la ecuación (14) hasta la ecuación (18).

Restando las filas uno y dos de (12) se obtienen las mismas expresiones (10) y (11), donde $m l$ es definida por (11). Restando las filas uno y tres de (12) se obtiene (14) y (15).

$$
R_{F(2)}=\frac{\operatorname{imag}\left\{\frac{V_{k a}^{f}-V_{k c}^{f}}{\Delta V_{a c}^{f}}\right\}}{\operatorname{imag}\left\{\frac{\Delta I_{F a c}^{f}}{\Delta V_{a c}^{f}}\right\}}
$$

$$
m_{2}=\frac{V_{k a}^{f}-V_{k c}^{f}-R_{F(2)} \Delta I_{F a c}^{f}}{\Delta V_{a c}^{f}}
$$

Donde,

$$
\begin{gathered}
\Delta V_{a c}^{f}=m\left(K_{4} I_{(k, F) a}^{f}+K_{5} I_{(k, F) b}^{f}+K_{6} I_{(k, F) c}^{f}\right)+R_{F} \Delta I_{F a c}^{f} \\
\Delta I_{F a c}^{f}=\left[\left(I_{(k, F) a}^{f}+I_{(k+1, F) a}^{f}\right)-\left(I_{(k, F) c}^{f}+I_{(k+1, F) c}^{f}\right)\right] \\
K_{4}=Z_{a a}-Z_{c a} \\
K_{5}=Z_{a b}-Z_{c b} \\
K_{6}=Z_{a c}-Z_{c c}
\end{gathered}
$$

Por último, restando las filas dos y tres de (12) se obtiene (17) y (18).

$$
\begin{gathered}
R_{F(3)}=\frac{\operatorname{imag}\left\{\frac{V_{k b}^{f}-V_{k c}^{f}}{\Delta V_{b c}^{f}}\right\}}{\operatorname{imag}\left\{\frac{\Delta I_{F b c}^{f}}{\Delta V_{b c}^{f}}\right\}} \\
m_{3}=\frac{V_{k b}^{f}-V_{k c}^{f}-R_{F(3)} \Delta I_{F b c}^{f}}{\Delta V_{b c}^{f}}
\end{gathered}
$$

Donde,

$$
\begin{gathered}
\Delta V_{b c}^{f}=m\left(K_{7} I_{(k, F) a}^{f}+K_{8} I_{(k, F) b}^{f}+K_{9} I_{(k, F) c}^{f}\right)+R_{F} \Delta I_{F b c}^{f} \\
\Delta I_{F b c}^{f}=\left[\left(I_{(k, F) b}^{f}+I_{(k+1, F) b}^{f}\right)-\left(I_{(k, F) c}^{f}+I_{(k+1, F) c}^{f}\right)\right] \\
K_{7}=Z_{b a}-Z_{c a} \\
K_{8}=Z_{b b}-Z_{c b} \\
K_{9}=Z_{b c}-Z_{c c}
\end{gathered}
$$

Después de obtenidas cada una de las distancias a la falla $m_{1}, m_{2}$ y $m_{3}$, se define la distancia a la falla como el promedio aritmético de las tres, menos la desviación estándar.

$$
\begin{gathered}
\bar{m}=\frac{\left(m_{1}+m_{2}+m_{3}\right)}{3} \\
s=\sqrt{\frac{\sum_{i=1}^{3}\left(m_{i}-\bar{m}\right)^{2}}{3}} \\
m=\bar{m}-s
\end{gathered}
$$

\section{ALGORITMO DE AJUSTE DE CORRIENTE DE FALLA PARA MÉTODO DE LOCALIZACIÓN DE FALLAS}

El algoritmo propuesto para la localización de fallas está orientado a la estimación de la corriente de falla por medio de las medidas de tensión y corriente de falla registradas en la GD. Aprovechando que se conoce el estado del sistema en falla, a partir de las medidas registradas en la subestación y en la GD, se puede conocer las corrientes y tensiones nodales en el radial donde se encuentra conectada esta fuente. El algoritmo asume que la falla se localiza en cualquiera sección del sistema, por lo tanto recorre cada sección asumiendo como corriente del nodo $(k+1)$ al nodo $F$ en falla, como la corriente en falla estimada desde la generación distribuida a la sección en estudio. El algoritmo generalizado para la estimación de la distancia a la falla se muestra en la Figura 5, y consta de las etapas descritas a continuación:

\section{Etapa 1: Extracción de información sobre la configuración del sistema}

La información del sistema se obtiene a partir de las bases de datos del operador de red. En este artículo se utiliza un sistema de potencia simulado en el 
software EMPT/ATP [14]. Adicionalmente se utiliza una herramienta conocida como ATPeXchange que permite extraer, organizar y procesar la información del sistema y almacenarla en un formato estándar [15].

Esta herramienta a su vez obtiene los radiales equivalentes del sistema de distribución, lo que permite una aplicación sencilla de los métodos de localización de fallas en cualquier sistema. Se entiende como sistema radial equivalente de un circuito al conjunto de tramos de línea que van desde el nodo inicial a un nodo final o a un nodo con GD, acumulando en los nodos de bifurcación las ramificaciones que se desprendan de este.

\section{Etapa 2: Estimación de las tensiones y corrientes en los nodos que conforman el radial GD}

La utilización de las medidas de tensión y corriente en la subestación del sistema y la GD representa una gran ventaja en la localización de fallas, ya que estas permiten el conocimiento del estado de operación del sistema en el instante de falla. Este conocimiento permite implementar una estrategia para considerar el efecto de la GD en la corriente de falla.

Para la definición de la estrategia se asume un alimentador de distribución como el mostrado en la Figura 1, el que tiene una falla entre el nodo $k$ y el $k+1$.

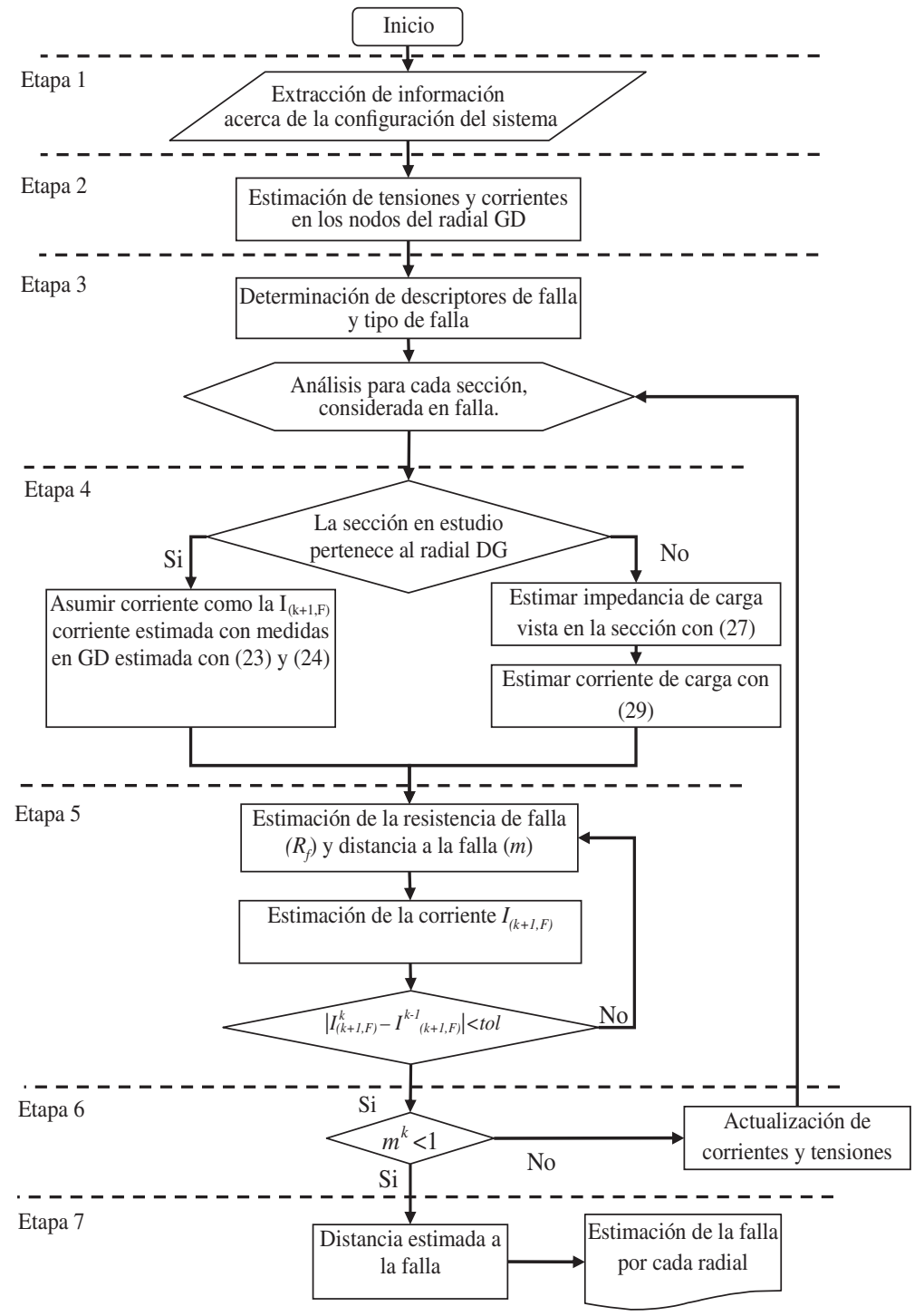

Figura 5. Algoritmo para la localización de fallas. 
Si se analiza de manera detallada el sistema se puede observar que la corriente $I_{(k+1, F)}^{f}$ en la sección en falla es la corriente obtenida en esta sección a partir de las medidas de tensión y corriente de falla del generador 2 (GD). No obstante, la localización de la falla no se conoce y es el principal objetivo de la metodología. Por tanto, asumiendo la configuración del sistema en estado de prefalla y utilizando las medidas en estado de falla de la GD, como se muestra en la Figura 6, se puede estimar las tensiones y corrientes en todos los nodos que conforman este radial utilizando las ecuaciones (23) y (24).

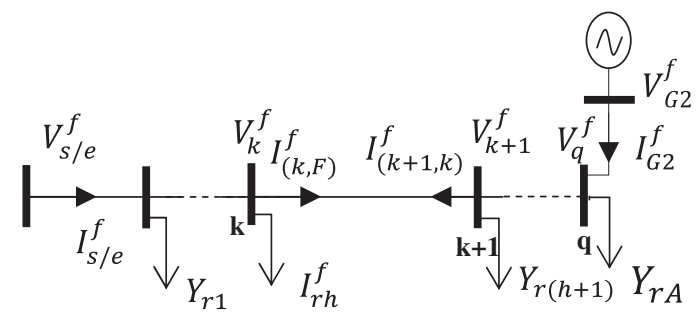

Figura 6. Modelo de sistema asumido para estimación de las tensiones y corrientes en los nodos que conforman el radial GD.

Las ecuaciones (23) y (24) definen los fasores de tensión y corriente en falla para el nodo $k+1$.

$$
\begin{gathered}
V_{(k+1)}^{f}=V_{G 2}^{f}-\sum_{i=1}^{k} Z_{i} \cdot I_{(i)}^{f} \forall k>1 \\
I_{(k+1, k)}^{f}=I_{G 2}^{f}-\sum_{i=1}^{k} Y_{r i} \cdot V_{(i+1)}^{f} \forall k>1
\end{gathered}
$$

Donde,

$Y_{r i}$ : Matriz de admitancia paralelo conectada al nodo $i$. Si no existe, se asume cero.

$Z_{i}$ : Matriz de impedancia de la línea de la sección $i$.

El algoritmo de localización mostrado en la Figura 5 recorre cada sección del sistema radial en estudio, por tanto para cada sección que pertenezca al radial GD la corriente $I_{(k+1, F)}^{f}$ se asume como la corriente $I_{(k+1, k)}^{f}$.

\section{Etapa 3: Determinación de descriptores de falla y tipo de falla}

Los descriptores de falla corresponden a los fasores de corriente y tensión en el estado de prefalla y falla. Estos descriptores se determinan por medio de los registros de falla medidos en la subestación y la generación distribuida [16]. El tipo de falla se determina por el algoritmo propuesto en [17], el que se aplica de manera individual con las medidas de tensión y corriente registradas en la subestación y las registradas en el punto de generación distribuida.

\section{Etapa 4: Estimación de la corriente $I_{(k+1, F)}^{f}$}

Para el sistema mostrado en la Figura 7 se pueden presentar dos condiciones bajo falla que determinan la manera de estimar la corriente $I_{(k+1, F)}^{f}$.

(a) Análisis de secciones en falla antes de la GD. Esta condición de falla se muestra en la Figura 7(a), donde la corriente $I_{(k+1, F)}^{f}$ se estima a partir de las medidas de tensión y corriente registradas en GD de la manera expuesta anteriormente.

(b) Análisis de secciones en falla antes de la GD. Esta condición de falla se muestra en la Figura 7(b), para ello, se observa que el efecto de la GD es considerado cuando se actualizan las tensiones y corrientes en el nodo donde se encuentra conectada esta fuente. Por tanto, la estimación de la corriente $I_{(k+1, F)}^{f}$ se realiza por medio de la impedancia de carga vista desde la sección en estudio y la tensión en el nodo de falla, con lo que la corriente $I_{(k+1, F)}^{f}$ está en función de la distancia a la falla $(m)$.

Para estimar la impedancia de carga vista desde cada nodo del sistema de potencia se asume el sistema en estado de prefalla. A partir de las tensiones y corrientes en la sección en análisis se estima la impedancia de falla como se muestra en la ecuación (25).

$$
\begin{aligned}
& Z_{l a_{k}}=\frac{V_{k_{a}}^{p}-\Delta V_{a}^{p}}{I_{(k, k+1)_{a}}^{p}} \\
& Z_{l b_{k}}=\frac{V_{k_{b}}^{p}-\Delta V_{b}^{p}}{I_{(k, k+1)_{b}}^{p}} \\
& Z_{l c_{k}}=\frac{V_{k_{c}}^{p}-\Delta V_{c}^{p}}{I_{(k, k+1)_{c}}^{p}}
\end{aligned}
$$




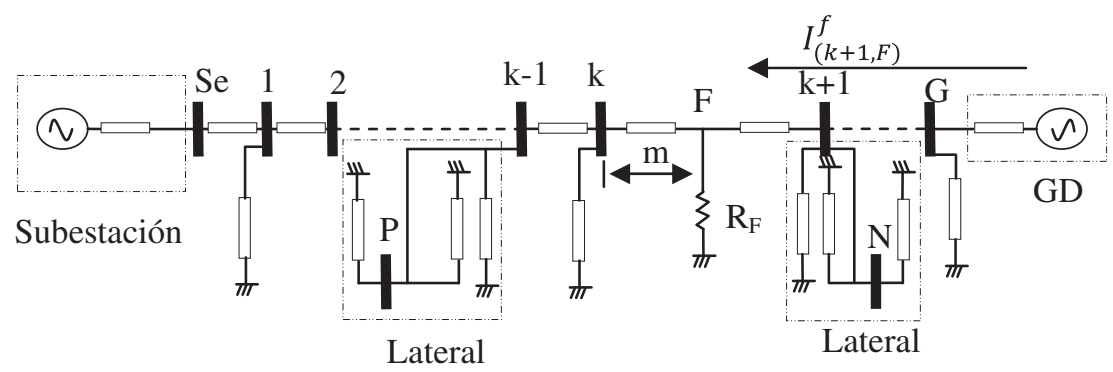

(a) Análisis para secciones en falla antes de la GD

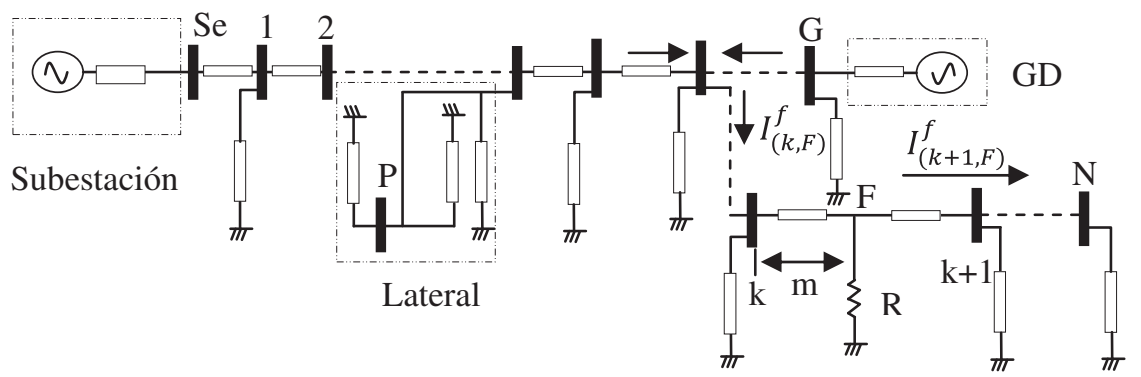

(b) Análisis para secciones en falla después de la GD

Figura. 7. Condiciones de análisis para las secciones en falla.

Con,

$\Delta V_{a}^{p}=Z_{a a} \cdot I_{(k, k+1)_{a}}^{p}+Z_{a b} \cdot I_{(k, k+1)_{b}}^{p}+Z_{a c} \cdot I_{(k, k+1)_{c}}^{p}$

$\Delta V_{b}^{p}=Z_{b a} \cdot I_{(k, k+1)_{a}}^{p}+Z_{b b} \cdot I_{(k, k+1)_{b}}^{p}+Z_{b c} \cdot I_{(k, k+1)_{c}}^{p}$

$\Delta V_{c}^{p}=Z_{c a} \cdot I_{(k, k+1) a}^{p}+Z_{c b} \cdot I_{(k, k+1) k_{b}}^{p}+Z_{c c} \cdot I_{(k, k+1)_{c}}^{p}$

La matriz de impedancia de carga vista desde cada nodo $k$ se define por (27).

$$
Z_{l k}=\left[\begin{array}{ccc}
Z_{l a_{k}} & 0 & 0 \\
0 & Z_{l b_{k}} & 0 \\
0 & 0 & Z_{l c_{k}}
\end{array}\right]
$$

Como la corriente $I_{(k+1, F)}^{f}$ depende de la distancia de falla y la impedancia de carga, se debe desarrollar un proceso iterativo para su cálculo. Este proceso se muestra a continuación.

Etapa 5: Proceso de refinamiento: estimación de la distancia a la falla, la resistencia de falla y actualización de la corriente $I_{(k+1, F)}^{f}$.

El ciclo propuesto para el cálculo de la distancia y la resistencia de falla se fundamenta en un proceso recursivo para la estimación de la corriente $I_{(k+1, F)}^{f}$, ya que esta última es una de las variables que más afecta la estimación de los parámetros para la localización de la falla.

El proceso iterativo comienza con la asignación de los valores iniciales de las variables no conocidas, en este caso la corriente $I_{(k+1, F)}^{f}$. Esta corriente se inicializa con la ecuación (28). Debido a que la metodología de localización de fallas se definió con la dirección de la corriente $I_{(k+1, F)}^{f}$ hacia el nodo de falla, la corriente de carga para esta condición de falla debe ser estimada como $-I_{(k+1, F)}^{f}$.

$\left[\begin{array}{c}I_{(k+1, F) a}^{f} \\ I_{(k+1, F) b}^{f} \\ I_{(k+1, F) c}^{f}\end{array}\right]=-\left[\begin{array}{ccc}Z_{l a_{k}} & 0 & 0 \\ 0 & Z_{l b_{k}} & 0 \\ 0 & 0 & Z_{l c_{k}}\end{array}\right]^{-1}\left[\begin{array}{c}V_{k a}^{p} \\ V_{k b}^{p} \\ V_{k c}^{p}\end{array}\right]$

Después de inicializada la corriente $I_{(k+1, F)}^{f}$ se calcula la resistencia de falla y la distancia a la falla de la manera expuesta en método de localización de fallas. Conocida la resistencia y la distancia a la falla se recalcula la corriente $I_{(k+1, F)}^{f}$ por medio de la ecuación (29). 


$$
\left[I_{(k+1, F)}^{f}\right]_{w}=-\left[\left(1-m_{w}\right)\left[Z_{k}\right]+\left[Z_{l k}\right]\right]^{-1}\left[V_{F}^{f}\right]_{w}
$$

Donde,

$\left[V_{F}^{f}\right] \quad$ : Tensión de falla en el punto de falla $\left[V_{F}\right]_{w} \quad$ en la iteración $w$. $\left[I_{(k+1, F)}^{f}\right]_{w} \begin{aligned} & : \text { Corriente de carga en estado de falla } \\ & \text { en la iteración } w .\end{aligned}$

Estimada la corriente $\left[I_{(k+1, F)}^{f}\right]_{w}$, se determina la diferencia entre la corriente hallada en la iteración anterior y la actual, a partir de la ecuación (30).

$$
\operatorname{Error}_{w}=\left|\left[I_{(k+1, F)}^{f}\right]_{w}-\left[I_{(k+1, F)}^{f}\right]_{w-1}\right|
$$

El error calculado corresponde al primer criterio de parada en el proceso iterativo. Este criterio define la calidad del refinamiento realizado a la distancia de falla a partir de la actualización de la corriente $I_{(k+1, F)}^{f}$. Cuando se cumple este criterio se observa si la distancia a la falla calculada es menor a uno (distancia normalizada); de ser así, se determina la distancia real estimada por el método. En caso contrario se actualizan corrientes y tensiones nodales y se continúa en el siguiente tramo.

Este proceso de refinamiento solo se realiza para la situación de falla (b). De lo contrario la distancia a la falla se estima directamente con la corriente de carga calculada en el paso anterior.

\section{Etapa 6: Actualización de corrientes y tensiones cuando el punto de falla se encuentra en un tramo diferente al analizado}

La actualización de corrientes y tensiones consiste en el cálculo de la tensión de recibo en el tramo actual, la que corresponde a la tensión de envío en el tramo siguiente. Este cálculo se realiza en prefalla y falla por medio de las expresiones (31) y (32), respectivamente.

$$
\begin{aligned}
& {\left[V_{(k+1)}^{p}\right]=\left[V_{(k)}^{p}\right]-\left[Z_{k}\right] \cdot\left[I_{(k, k+1)}^{p}\right]} \\
& {\left[V_{(k+1)}^{f}\right]=\left[V_{(k)}^{f}\right]-\left[Z_{k}\right] \cdot\left[I_{(k, k+1)}^{f}\right]}
\end{aligned}
$$

La actualización de la corriente se realiza utilizando la admitancia nominal por cada ramal, donde se calcula la corriente para la siguiente sección utilizando los valores de tensión y corriente de la sección actual, como se muestra en las ecuaciones (33) y (34).

$$
\begin{gathered}
{\left[I_{(k+1, k+2)}^{p}\right]=\left[I_{(k, k+1)}^{p}\right]-\left[Y_{r h}\right] \cdot\left[V_{(k+1)}^{p}\right]} \\
{\left[I_{(k+1, F)}^{f}\right]=\left[I_{(k, F)}^{f}\right]-\left[Y_{r h}\right] \cdot\left[V_{(k+1)}^{f}\right]}
\end{gathered}
$$

No obstante, se debe tener especial cuidado en el momento de actualizar la corriente en el nodo donde se encuentra conectado el ramal con GD, ya que se debe considerar el aporte de corriente de esta fuente. Por tanto, para este caso las expresiones (33) y (34) deben ser redefinidas como se muestra en (35) y (36).

$\left[I_{(k+1, k+2)}^{p}\right]=\left[I_{(k, k+1)}^{p}\right]+\left[I_{(g, k)}^{f}\right]-\left[Y_{r h}\right] \cdot\left[V_{(k+1)}^{p}\right](35)$

$\left[I_{(k+1, F)}^{f}\right]=\left[I_{(k, F)}^{f}\right]+\left[I_{(g, k)}^{p}\right]-\left[Y_{r h}\right] \cdot\left[V_{(k+1)}^{f}\right]$

Donde $\left[I_{(g, k)}^{p}\right]$ es la corriente en estado de prefalla del nodo $g$ al nodo $k$.

\section{Etapa 7: Obtención de verdadera distancia estimada}

Después de localizada la falla se determina la distancia real desde la subestación, realizando la sumatoria de las distancias de las secciones recorridas por el algoritmo y adicionándole la porción del tramo donde se localiza la falla por medio del último valor obtenido de " $m$ " (37).

$$
L_{\text {est }}=\sum_{i=1}^{n t-1} L_{\text {tramo }_{i}}+m_{\text {ult }} \cdot L_{\text {tramo }_{n t}}
$$

Donde,

$L_{e s t} \quad$ : Distancia estimada desde la subestación al punto donde se localiza la falla.

nt : Número de tramos analizados.

$m_{\text {últ }} \quad$ : Última distancia a la falla estimada en el tramo $n t$.

$L_{\text {tramo } 1}$ : Longitud del último tramo analizado.

\section{PRUEBAS Y RESULTADOS}

El sistema seleccionado para la validación de la metodología propuesta es el sistema IEEE 34 nodos, este es tomado de los "test feeders" del "Distribution System Analysis Subcommittee" del "Institute Electrical and Electronics Enginieers" [18]. Este alimentador está ubicado en el estado de Arizona (Estados Unidos) y opera a una tensión de 24,9 kV. Entre sus principales características están 
la presencia de ramales monofásicos, bifásicos y trifásicos, múltiples calibres de conductor, cargas desbalanceadas de naturaleza concentrada y distribuida. Este sistema se modela en el software de simulación ATPDraw y se modifica introduciendo un generador distribuido.

Adicionalmente se conecta una impedancia de Thevenin a la GD que permite variar la corriente aportada al circuito y de esta manera simular diferentes niveles de penetración de generación distribuida en el sistema. Sobre este sistema se simularon fallas monofásicas, bifásicas y trifásicas, a resistencias de falla de $0 \Omega, 20 \Omega$ y $40 \Omega$ con un nivel de penetración de generación distribuida de $5 \%$ a 50\%. La Figura 8 muestra el sistema IEEE 34 en el entorno del ATPDraw.

El nivel de penetración de generación distribuida (\%GD) es calculado a partir de la ecuación (38).

$$
\% D G=\left(\frac{I_{D G}}{I_{s / e}+I_{D G}}\right) \times 100 \%
$$

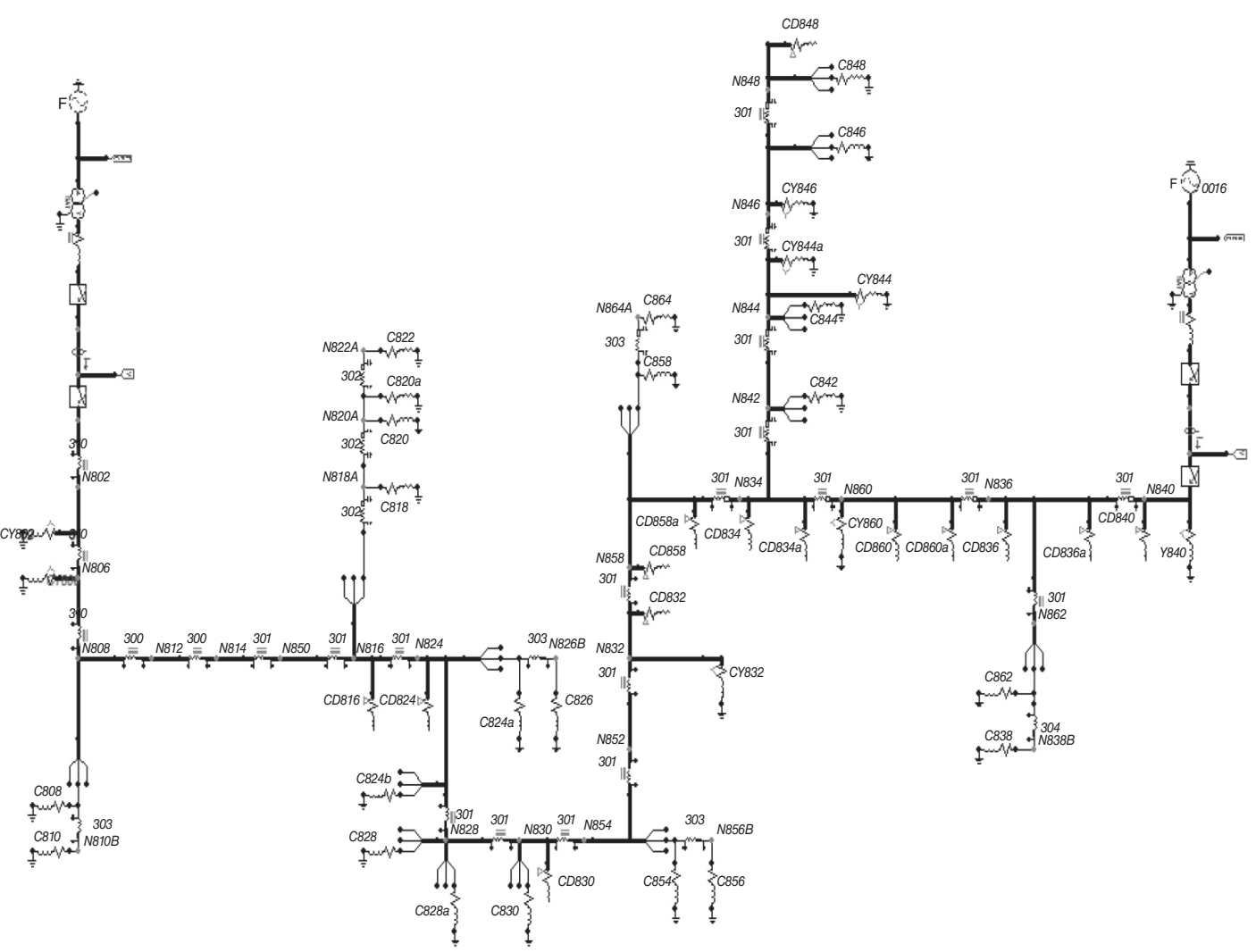

Donde,

$I_{G D}$ : Corriente de la GD en estado nominal.

$I_{s / e}:$ Corriente de la subestación en estado nominal.

Los resultados se muestran a partir de superficies de desempeño, donde se presenta el error de la distancia calculada contra la distancia real de la falla respecto del nivel de penetración de GD. El error se calcula a partir de la ecuación (39).

$$
\text { error }=\frac{\left|d_{\text {estimada }}-d_{\text {real }}\right|}{d_{\text {total }}}
$$

Donde,

$d_{\text {estimada }}$ : Distancia a la falla estimada por la estrategia propuesta.

$d_{\text {real }} \quad$ : Distancia real al punto de falla.

$d_{\text {total }} \quad$ : Distancia total del radial equivalente donde ocurrió la falla.

Los resultados para las fallas monofásicas se presentan en la Figura 9.

Figura 8. Sistema IEEE 34 en ATPDraw. 


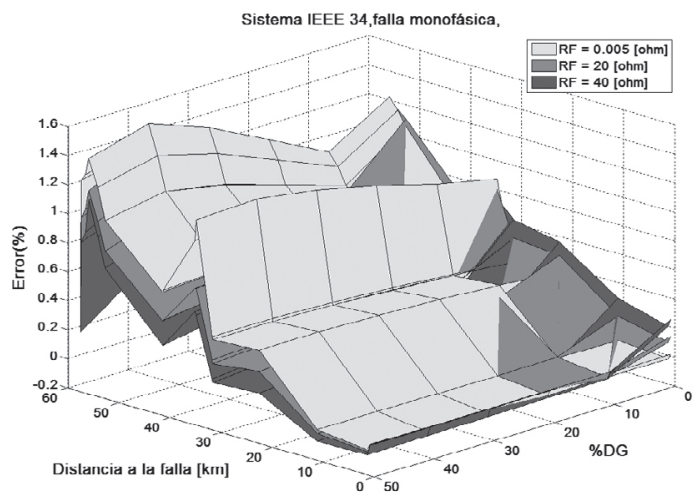

Figura 9. Superficies de desempeño, falla monofásica.

De la Figura 9 se puede observar una clara tendencia a la sobreestimación de la distancia de falla. Igualmente se puede notar un leve incremento en el error de estimación ante la variación del nivel de penetración de la GD para cada uno de los tres valores de resistencia de falla. No obstante, el desempeño del método es sobresaliente a pesar que los incrementos en la GD fueron significativamente grandes, llegándose a presentar un nivel de penetración igual al de la fuente principal ( $\% \mathrm{GD}=50$ ).

La Figura 10 presenta los resultados para las fallas bifásicas, donde se muestran las superficies de desempeño enfrentan el error de estimación contra distancia real, respecto del nivel de penetración de la GD. Además, se obtienen tres superficies que corresponden a tres valores de resistencia de falla diferentes $(0,20$ y $40 \Omega)$.

El error de estimación máximo presente para las fallas bifásicas es de -1,5\%, no obstante, su comportamiento es de subestimación de la distancia a la falla. Además, el efecto de la GD es más notable que en la falla monofásica. También se puede observar un mayor efecto en el error con el aumento de la resistencia de falla, respecto de la falla monofásica.

Por último, la Figura 11 presenta los resultados para las fallas trifásicas.

El desempeño de la metodología para las fallas trifásicas es muy similar al de las fallas bifásicas. Su comportamiento es de subestimación de la distancia a la falla, presentándose un error máximo de $-2 \%$. Igual que para los otros tipos de fallas, el aumento del nivel de penetración de la GD afecta su

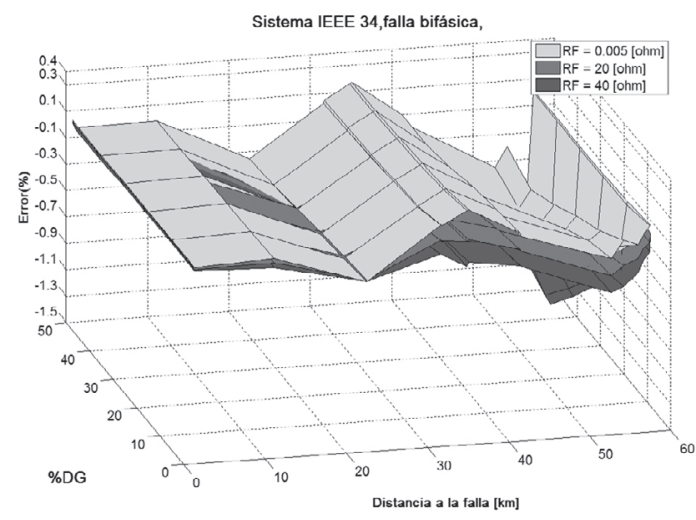

Figura 10. Superficies de desempeño, falla bifásica.

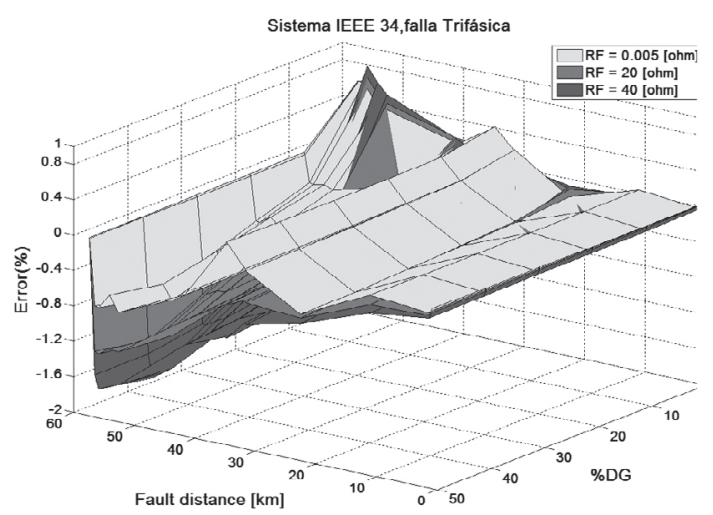

Figura 11. Superficies de desempeño, falla trifásica.

desempeño, aunque sigue siendo un error aceptable de estimación para los casos analizados. Adicionalmente la resistencia de falla afecta levemente el desempeño de la metodología.

\section{CONCLUSIONES}

La metodología de localización de fallas propuesta permite considerar de manera exitosa el efecto de la generación distribuida por medio de la estimación de la corriente de falla a partir de las medidas de tensión y corriente registradas en la GD.

Este aporte permite extender de manera simple cualquier metodología de localización de fallas basada en la estimación de la impedancia aparente, para sistemas de distribución con GD.

Además, la utilización de las medidas de tensión y corriente en la GD evita la utilización del modelo del generador y por tanto sus parámetros, que en la mayoría de los casos no se conocen con precisión. 
Esta característica hace que sea una metodología muy práctica y de fácil aplicación en sistemas reales.

Por último, la metodología demostró ser robusta, siendo validada en un sistema de distribución exigente, el que fue modelado lo más cercano posible a la realidad y en los escenarios de validación más críticos que se pueden presentar ante un evento de falla (resistencias de fallas entre 0 y 40 y diferentes porcentajes de GD [5\%-50\%]).

El mayor error de estimación presente ocurre para fallas trifásicas, siendo de $-2 \%$, esto es un error aceptable y respalda la validez de la metodología propuesta.

\section{AGRADECIMIENTOS}

Este trabajo fue desarrollado en el grupo de investigación en calidad de la energía eléctrica y estabilidad ICE3 de la Universidad Tecnológica de Pereira. Para su desarrollo se recibió apoyo de la Vicerrectoría de Investigaciones, Innovación de la Universidad Tecnológica de Pereira y de Colciencias por medio del proyecto de investigación "Desarrollo de localizadores robustos de fallas paralelas de baja impedancia para sistemas de distribución de energía eléctrica -LOFADIS2012-", contrato 0977-2012.

\section{REFERENCIAS}

[1] M. Kezunovic. "Smart Fault Location for Smart Grids". IEEE Trans. Smart Grid. Vol. $2 \mathrm{~N}^{\circ}$ 1, pp. 11-22. March, 2011.

[2] K. Kauhaniemi and L Kumpulainen. "Impact of distributed generation on the protection of distribution networks". Dev. Power Syst. Prot. Vol. 1 No 0537-9989, pp. 315-318. 2004.

[3] G. Morales-Espana, J. Mora-Florez and H. Vargas-Torres. "Fault location method based on the determination of the minimum fault reactance for uncertainty loaded and unbalanced power distribution systems". In 2010 IEEE/PES Transmission and Distribution Conference and Exposition: Latin America (T\&D-LA), pp. 803-809. 2010.

[4] R.H. Salim, K.C.O. Salim and A.S. Bretas. "Further improvements on impedance-based fault location for power distribution systems".
IET Gener. Transm. Distrib. Vol. $5 \mathrm{~N}^{\mathrm{o}} 4$, p. 467. 2011.

[5] M.-S. Choi, S.-J. Lee, S.-I. Lim, D.-S. Lee and X. Yang. "A Direct Three-Phase Circuit Analysis-Based Fault Location for Lineto-Line Fault". IEEE Trans. Power Deliv. Vol. $22 \mathrm{~N}^{\circ}$ 4, pp. 2541-2547. October, 2007.

[6] M. Choi, S. Lee, D. Lee and B. Jin. "Analysis for Distribution Systems". IEEE Trans. Power Deliv. Vol. 19 No 1, pp. 35-41. 2004.

[7] A. Bretas and K.C.O. Salim. "Fault Location in Unbalanced DG Systems using the Positive Sequence Apparent Impedance". In Transmission \& Distribution Conference and Exposition: Latin America. 2006. TDC '06. IEEE/PES, pp. 1-6. 2006.

[8] R.H. Salim. "Novos desenvolvimentos na localização de faltas em sistemas de distribuição de energia elétrica fundamentada na impedância”. Tesis para optar al grado de Magister. Universidade Federal do Rio Grande do Sul. 2008.

[9] J.U.N. Nunes, S. Member and A.S. Bretas. "Impedance-Based Fault Location Formulation for Unbalanced Primary Distribution Systems with Distributed Generation”. 2010.

[10] J.U.N. De Nunes and A.S. Bretas. "An Extended Fault Location Formulation for Unbalanced Distribution Feeders with Distributed Generation". No 1.2010.

[11] J.I. Marvik, A. Petterteig and H.K. Hoidalen. "Analysis of Fault Detection and Location in Medium Voltage Radial Networks with Distributed Generation". 2007 IEEE Lausanne Power Tech, pp. 1191-1196. July, 2007.

[12] H.K. Marvik, J. Hidalen and A. Petterteig, "Localization of short-circuits on a medium voltage feeder with distributed generation". In 20th International Conference on Electricity Distribution. No 0743, pp. 8-11. 2009.

[13] G. Morales-Espana, J. Mora-Florez and H. Vargas-Torres. "Elimination of Multiple Estimation for Fault Location in Radial Power Systems by Using Fundamental Single-End Measurements". IEEE Trans. Power Deliv. Vol. 24 No 3, pp. 1382-1389. July, 2009.

[14] L. Prikler and H.K. Høidalen. "ATPDRAW for Windows 9x / NT / 2000 / XP Users' Manual". 2002. 
[15] A. Bedoya, J. Mora and S. Pérez. "Estrategia de reducción para la aplicación generalizada de localizadores de fallas en sistemas de distribución de energía eléctrica". Rev. EIA, pp. 21-37. 2012.

[16] J.J.M. Flórez. "Localización de faltas en sistemas de distribución de energía eléctrica usando métodos basados en el modelo y métodos basados en el conocimiento". Universitat de Girona. ISBN: 9788469045138. 2007.

[17] R. Das. "Determining the locations of faults in distribution systems". University of Saskatchewan. 1998.

[18] Distribution System Analysis Subcommittee. “IEEE 34 Node Test Feeder". 2001. 